\title{
Parameter-Adaptive VMD Method Based on BAS Optimization Algorithm for Incipient Bearing Fault Diagnosis
}

\author{
Heng-di Wang $\mathbb{D},{ }^{1}$ Si-er Deng $\mathbb{D},{ }^{1}$ Jian-xi Yang, ${ }^{1}$ Hui Liao, ${ }^{2}$ and Wen-bo Li ${ }^{1}$ \\ ${ }^{1}$ School of Mechatronics Engineering, Henan University of Science and Technology, Luoyang, Henan 471003, China \\ ${ }^{2}$ School of Mechanical Engineering, Northwestern Polytechnical University, Xi'an, Shanxi 710072, China \\ Correspondence should be addressed to Si-er Deng; dse@haust.edu.cn
}

Received 16 July 2019; Revised 29 December 2019; Accepted 27 January 2020; Published 25 February 2020

Academic Editor: Cedrick A. K. Kwuimy

Copyright $\odot 2020$ Heng-di Wang et al. This is an open access article distributed under the Creative Commons Attribution License, which permits unrestricted use, distribution, and reproduction in any medium, provided the original work is properly cited.

In view of the incipient fault characteristics are difficult to be extracted from the raw bearing fault signals, an incipient bearing fault diagnosis method based on parameter-adaptive variational mode decomposition (VMD) is proposed. The beetle antennae search (BAS) algorithm is adopted to seek for the optimal combination of the VMD parameters. The reciprocals of the calculated kurtosis values of intrinsic mode functions (IMFs) decomposed via VMD are employed as a fitness function in the searching process. The optimal mode number and the quadratic penalty term of VMD are adaptively set after the search. Afterwards, a vibration signal is decomposed into a set of IMFs using the parameter-adaptive VMD, and the IMF with the maximal kurtosis value is selected as the sensitive one. The selected IMF is further analyzed by Hilbert envelope demodulation. The resulting envelope spectrum can show the significant fault impulse characteristics which are highly helpful to diagnose incipient bearing faults. The kurtosis and the proportion of fault energy are introduced as the input vector of the extreme learning machine (ELM). Comparisons have been conducted via ELM to evaluate the performance by using EMD and the fixed-parameter VMD. The experimental results demonstrate that the proposed method is more effective in extracting the incipient bearing fault characteristics.

\section{Introduction}

Bearing is one of the most critical components in rotating machinery. The presence of defects in the bearing may lead to noise, vibration, or even system breakdown. For this reason, the bearing fault diagnosis has received considerable attention in the past decades.

It has become a widely acceptable method to assess the bearing performance by studying the vibration signals. As a result, this method has been adopted in many fields, such as bearing fault diagnosis [1-3], performance evaluation $[4,5]$, and equipment health monitoring [6-8].

However, the bearing vibration signals are ordinarily nonstationary and may be polluted by noise interference $[6,9]$. Additionally, the measured fault characteristic frequencies usually could not exactly match the theoretically values due to geometry imperfection, speed variation, or load variation [10], and so on. All of these factors perplex the extraction of fault characteristics from the raw signals.
Bearing fault diagnosis is mainly applied in two fields which are the quality control in bearing manufactories and the condition monitoring of the bearing in service, respectively. This paper is based on the former application and focuses on the fault diagnosis techniques. In this case, the bearing faults are mostly characterized as incipient faults which mean that the faults are slight or tiny, and the characteristics are not obvious. It gets difficult to accurately recognize and diagnose the incipient faults. At the same time, the measurements suffer little negative influence introduced by other mechanical transmission parts such as gears. Bearing manufactories usually complete bearing vibration measurement on the dedicated vibration tester. The measurement conditions are that the outer ring of the bearing is stationary, the inner ring rotates together with the shaft at the specified speed, and the specified load is applied at the same time.

Empirical mode decomposition (EMD) $[11,12]$ is a wellknown adaptive time-frequency processing method and can 
recursively decompose signals without the prior knowledge. A large number of scholarly articles covering a variety of applications have been published. However, EMD suffers from some shortcomings, such as the limited mathematical understanding, mode mixing, pseudocomponents, and end effects [9].

Variational mode decomposition (VMD) is a novel and self-adaptive signal decomposition algorithm proposed by Dragomiretskiy and Zosso in 2014 [13]. In comparison with the recursive EMD method, VMD can decompose a signal into an ensemble of band-limited intrinsic mode functions (IMFs) synchronously. The literature [13-15] compared the performance of VMD and EMD and deduced that VMD outperformed EMD with regard to the noise robustness and the effectiveness of feature extraction.

However, a significant drawback of VMD is that the parameters, namely, the mode number $M$ and the quadratic penalty term $\alpha$, need to be set in advance. Unreasonable preset parameters may induce the loss of effective modes or the mixing of different components [16] and affect the subsequent feature extraction results. $M$ should be specified based on the number of different frequency components contained in the raw signals, while $\alpha$, namely, the mode frequency bandwidth control parameter, is determined based on each central frequency of the mode. They are correlated with each other. A large number of modes may result in redundant VMD information. Correspondingly, a small number of modes may lead to mode mixing in the VMD results. $\alpha$ is related to the performance of suppressing noise interference. As $\alpha$ decreases, the bandwidth of the mode tends to be wide, and the VMD results may involve more background noise. But, the VMD results are likely to be distorted if the bandwidth is too narrow [14]. Since the two parameters need to be specified in advance without any prior knowledge about the raw signals, it is difficult to guarantee the accuracy of the VMD results. Accordingly, seeking for the optimum of $M$ and $\alpha$ that matches with the analyzed signals is the key to the VMD method.

In order to overcome the critical drawback, some studies have been conducted. Lian et al. [17] chose $M$ according to a series of indicators including permutation entropy, extreme value in the frequency domain, kurtosis criterion, and energy loss coefficient. Liu et al. [18] estimated $M$ by using the minimum redundancy maximum relevance. The above two methods in $[17,18]$ both optimized the value of $M$ but neglected the influence of $\alpha$ on decomposition results. Based on the grasshopper optimization algorithm, Zhang et al. [19] proposed a method to obtain the optimum of $M$ and $\alpha$. Wang et al. [20] used multi-objective particle swarm optimization (MOPSO) to obtain the optimum of $M$ and $\alpha$, in which the symbol dynamic entropy and the power spectral entropy were selected as the objective function of MOPSO. In [16], an improved adaptive genetic algorithm was proposed to optimize the two parameters of VMD. The performance of several decomposition methods for the simulation signal was compared, and authors considered that the decomposition effect of VMD preceded those of other methods. In [19], the effective value range of $\alpha$ was preassigned in the interval of $[1000,10,000]$. However, with separate optimization algorithm, our experimental results described in the following as well as the literature [16] indicate that the optimal $\alpha$ may lie out of the interval stated in [19], respectively.

Compared with the above key parameters, the noise tolerance $\tau$ and the convergence tolerance level $\varepsilon$ have little influence on the decomposition results; thus, the default values in the original VMD method are usually adopted [19].

In this paper, a novel optimization algorithm called beetle antennae search (BAS) is employed to estimate the optimal $M$ and $\alpha$. BAS is a nature-inspired algorithm developed by Jiang and $\mathrm{Li}$ [21]. In [21], the global optimization performance of BAS was benchmarked on the Michalewicz function and the Goldstein-Price function, in which the numerical results validated the efficacy of this algorithm.

\section{BAS Principles}

BAS is a nature-inspired algorithm to solve the optimization problems, which mimics the detecting and searching behaviors of long-horn beetles. A beetle wobbles its two antennae to detect the odour while preying or finding mates, i.e., the beetle explores nearby area randomly using a pair of antennae. When the antenna in one side receives a higher concentration of odour, the beetle would turn to the direction towards the same side; otherwise, it would turn to the other side. Searching behavior of beetles may be formulated in a way which is associated with an objective function to be optimized.

The position of the beetle can be expressed as a vector $\mathbf{x}^{t}$ at $t$-th time instant $(t=1,2, \ldots) . f(\mathbf{x})$ is denoted as a fitness function which indicates the concentration of odour at position $\mathbf{x}$. The maximal value of $f(\mathbf{x})$ corresponds to the source point of the odour, namely, the optimal solution of the function.

The random direction of searching behavior can be modeled as follows:

$$
\overrightarrow{\mathbf{b}}=\frac{\operatorname{rnd}(c, 1)}{\|\operatorname{rnd}(c, 1)\|},
$$

where $\operatorname{rnd}(\cdot)$ presents a random function and $c$ denotes the dimensions of the position. The barycenter coordinates of both right-hand and left-hand antennae can be then described in the following equation:

$$
\begin{aligned}
& \mathbf{x}_{r}=\mathbf{x}^{t}+s^{t} \overrightarrow{\mathbf{b}}, \\
& \mathbf{x}_{l}=\mathbf{x}^{t}-s^{t} \overrightarrow{\mathbf{b}},
\end{aligned}
$$

where $\mathbf{x}_{r}$ and $\mathbf{x}_{l}$ stand for the barycenter coordinate of the right-hand antenna and that of the left-hand antenna, respectively, and $s$ is the sensing length. The value of $s$ should be large enough to cover an appropriate searching area for fear of falling into local minimum points at the beginning and then attenuate with the iterations.

Considering the searching behavior, the iterative model of detecting behavior can be formulated as follows:

$$
\mathbf{x}^{t}=\mathbf{x}^{t-1}+\delta^{t} \overrightarrow{\mathbf{b}} \operatorname{sign}\left(f\left(\mathbf{x}_{r}\right)-f\left(\mathbf{x}_{l}\right)\right)
$$


where $\operatorname{sign}(\cdot)$ represents a sign function and $\delta$ is the step size of searching. In consideration of convergence speed, $\delta$ follows a decreasing function of $t$. The initialization of $\delta$ should be equivalent to the sensing length $s$. As examples, the sensing length and the step size may be updated as follows:

$$
\begin{aligned}
& s^{t}=0.95 s^{t-1}+0.01, \\
& \delta^{t}=0.95 \delta^{t-1} .
\end{aligned}
$$

As aforementioned, the BAS algorithm can achieve efficient optimization without the acquisition of functional expression or the gradient of function. Compared with the particle swarm optimization algorithm [22, 23], the BAS algorithm only employs one individual, i.e., one beetle; thus, the computation is reduced.

\section{Parameter-Adaptive VMD Method Based on BAS}

In this section, a parameter-adaptive VMD method based on BAS for incipient bearing fault diagnosis is introduced. The basic idea of the proposed method is to seek for the optimal $M$ and $\alpha$ by using the BAS algorithm.

Kurtosis criterion [24-26] has been developed based on the resonance demodulation technique. As a dimensionless index, the kurtosis can be used as a measure of impact components in the signals. The impact impulses caused by defect will increase the kurtosis obviously. Dong [27] introduced the spectral $L p / L q$ norm and deduced that the kurtosis could be explained as a special case of the spectral $L p / L q$ norm. Dong drew a conclusion that the spectral $L p / L q$ norm could be used for characterizing the repetitive impulses in the bearing vibration signals.

The bearing faults, in view of the application described in this paper, are mostly characterized by surface defects such as pitting and spot, which account for more than $90 \%$ of all faults. In our experiments, the kurtosis is effective for the diagnosis of bearing faults and can be used to identify the conditions of the bearings. In consideration of this advantage, the reciprocal of kurtosis values calculated from modes may be adopted as the fitness function to optimize VMD parameters. The optimization objective is to search for the mode with the maximal kurtosis, which indicates the most obvious fault information is contained in the screening mode.

$$
\begin{aligned}
f(\mathbf{x}) & =\min _{\{M, \alpha\}}\left\{\frac{1}{K_{i}}\right\}, \\
\text { s.t. } M & =3,4, \ldots, 8 .
\end{aligned}
$$

After obtaining the optimal parameters of VMD, the vibration signals are decomposed into a set of IMFs via VMD, and the mode with the maximal kurtosis is selected as the sensitive one. The selected mode may be further analyzed by Hilbert envelope demodulation to estimate whether the bearing is defective.

The following steps detail the procedures of the proposed fault diagnosis method:
Step 1: calculate the fault characteristic frequencies according to the geometry of the bearing and its rotational speed.

Step 2: in order to optimize $M$ and $\alpha$, initialize the vector $\mathbf{x}^{t}=\left\{M_{1}, \alpha_{1}\right\}$. In this paper, $M$ is preset as an integer in the interval of $[3,8]$, and the initial values of $M_{1}$ and $\alpha_{1}$ are equal to 3 and 2000, respectively.

Step 3: with each $\mathbf{x}$, decompose the vibration signals into a set of IMFs via VMD. Compute the kurtosis values of all IMFs, and specify the reciprocal of the maximal kurtosis as the value of $f(\mathbf{x})$.

Step 4: model the normalized random searching direction $\overrightarrow{\mathbf{b}}$ according to equation (1). Calculate $\mathbf{x}_{r}$ and $\mathbf{x}_{l}$ with the aid of equation (2), where $\mathbf{x}_{r}^{t}=\left\{M_{r}, \alpha_{r}\right\}$ and $\mathbf{x}_{l}^{t}=\left\{M_{l}, \alpha_{l}\right\}$.

Step 5: decompose the vibration signals via VMD by using $\mathbf{x}_{r}$ and $\mathbf{x}_{l}$, separately. And then calculate the respective $f\left(\mathbf{x}_{r}\right)$ and $f\left(\mathbf{x}_{l}\right)$.

Step 6: update $\mathbf{x}^{t}$ by solving equation (3).

Step 7: reiterate Steps 4-6 through 100 loops to acquire the optimal $M$ and $\alpha$.

Step 8: decompose the vibration signals into a set of IMF components via VMD by using the optimal $M$ and $\alpha$, and choose the IMF with the maximal kurtosis.

The flowchart of the proposed method is illustrated in Figure 1.

The proposed method is applied to the low-noise deep groove ball bearing 6203. The geometry of bearing 6203 is listed in Table 1. More details about the calculation of the fault characteristic frequencies can be found in [28]. The measurement conditions are in accordance with those described in Section 1.

\section{Applications on Bearing Fault Diagnosis}

The experimental setup is a self-developed bearing vibration tester, as shown in Figure 2. Vibration signals are sampled via two vibration velocity sensors. A data acquisition card PCI-6143 is used to sample the conditioned vibration signals. The sampling rate and the sampling numbers are set to $45 \mathrm{KHz}$ and 8192, respectively. The spindle speed is $1800 \mathrm{r} /$ $\min$.

4.1. Incipient Inner Raceway Fault. Figure 3 shows a timedomain vibration signal of bearing 6203 with the incipient inner raceway fault. As shown, it is difficult to find periodic impact impulses in the time-domain waveform.

The above vibration signal is processed by the proposed method. $\tau$ and $\varepsilon$ in the VMD method are both set to the default values, i.e., $\tau=0$ and $\varepsilon=1 \times 10^{-7}$. The optimal $M$ and $\alpha$ acquired by BAS are 8 and 380, respectively. Decomposition results of the signal via VMD with the optimal parameters are presented in Figure 4. The kurtosis values $(K)$ of IMFs are calculated and listed in Table 2. The maximal kurtosis appears in IMF3, namely, the IMF3 has the most observable impact composition. 


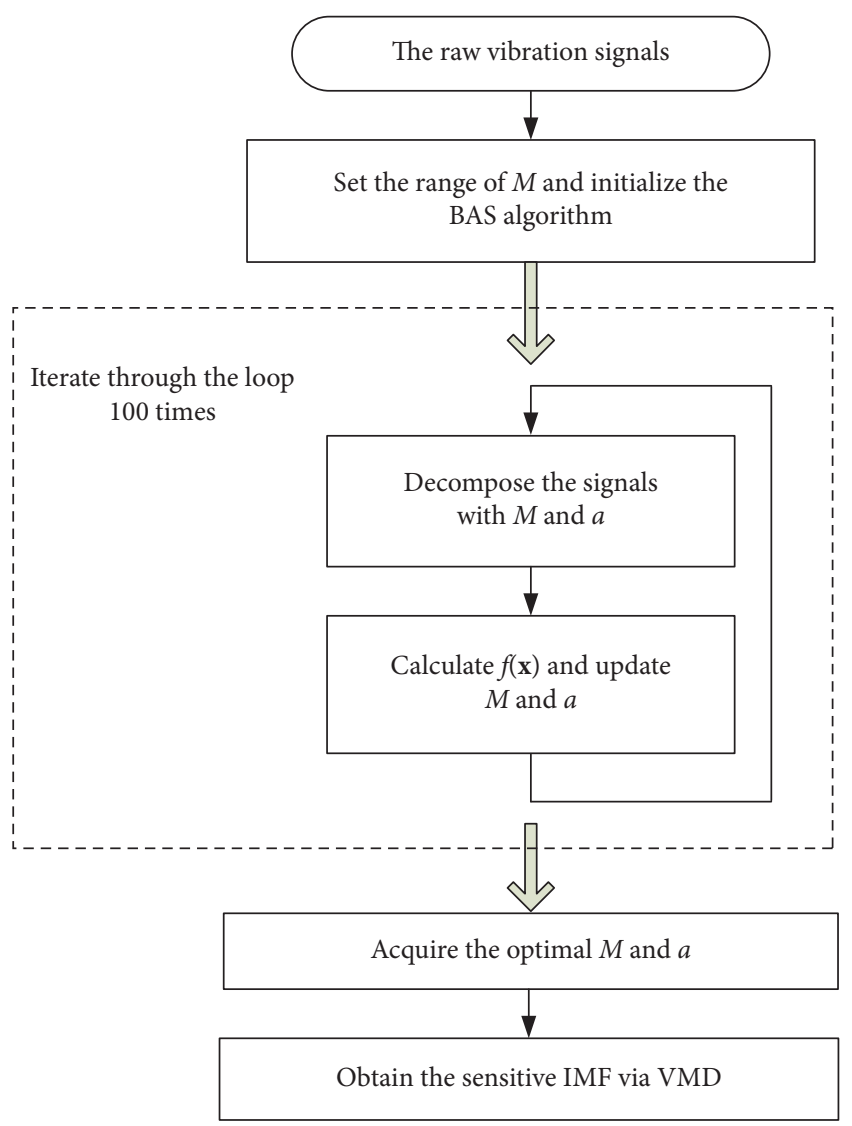

FIGURE 1: Flowchart of the proposed method.

TABLE 1: Information of the experimental bearing 6203.

\begin{tabular}{lc}
\hline Geometric parameters & \\
Outer race diameter, $d_{o}$ & $40 \mathrm{~mm}$ \\
Inner race diameter, $d_{i}$ & $17 \mathrm{~mm}$ \\
Rolling element diameter, $d$ & $6.747 \mathrm{~mm}$ \\
Number of the rolling elements, $z$ & 8 \\
Pitch diameter, $D$ & $29 \mathrm{~mm}$ \\
Contact angle, $\theta$ & $0^{\circ}$ \\
Spindle rotational frequency, $f_{s}$ & $30 \mathrm{~Hz}$ \\
\hline Fault characteristic frequencies & \\
Inner raceway, $f_{i}$ & $148 \mathrm{~Hz}$ \\
Outer raceway, $f_{o}$ & $92 \mathrm{~Hz}$ \\
Rolling element, $f_{b}$ & $122 \mathrm{~Hz}$ \\
\hline
\end{tabular}

As shown in Figure 4, despite IMF3 is still polluted by a little noise, significant time-domain impulses can be found. For further evaluating the fault characteristic frequency of the inner raceway, IMF3 is demodulated by Hilbert envelope analysis. The consequent envelope spectrum is shown in Figure 5.

It can be observed that after processed by the proposed method, 5 spectrum peaks are highlighted in the envelope spectrum of IMF3. These 5 spectrum peaks correspond to the fault characteristic frequency $f_{i}(148.3 \mathrm{~Hz})$ and its harmonics. The evident features suggest the inner raceway of the bearing is defective.

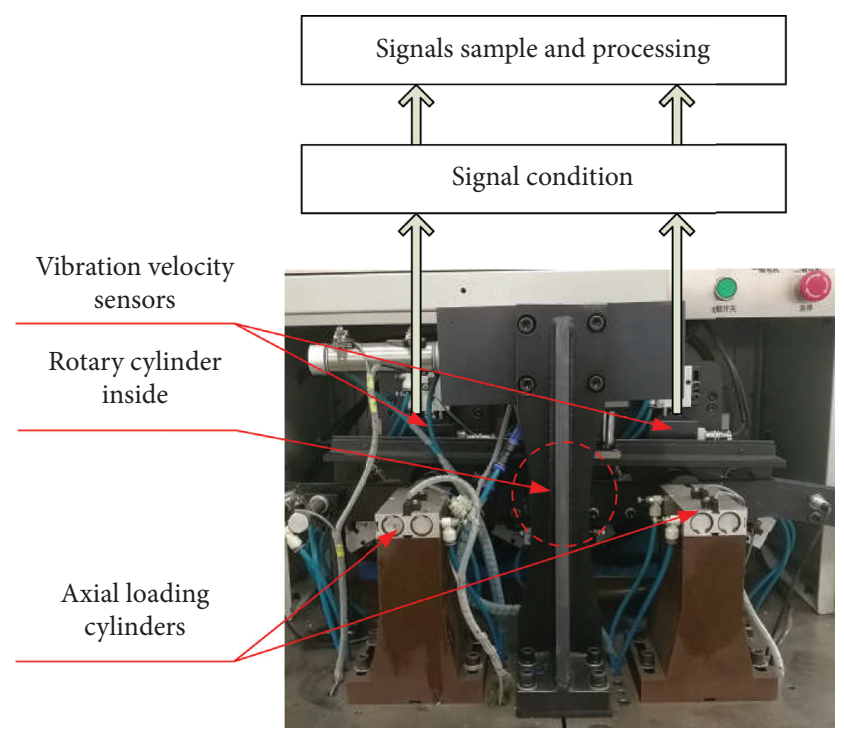

FIGURE 2: Sketch of the bearing vibration tester.

For the sake of comparison, the signal shown in Figure 3 is decomposed by means of EMD. The results are illustrated in Figure 6. As shown, faint time-domain impulses can be found in mode 4 and mode3. The kurtosis values of the modes are calculated and listed in Table 3.

Similarly, mode4 with the maximal kurtosis value is chosen and demodulated by Hilbert envelope analysis. The corresponding envelope spectrum is shown in Figure 7.

In order to further validate the effectiveness of the proposed method in extracting the bearing fault characteristics, the fixed-parameter VMD method is adopted to process the signal shown in Figure 3. Drawing on the experiences in the literature [15], $M$ is set to 4 , while $\alpha$, $\tau$, and $\varepsilon$ are all set to the default values in original VMD, i.e., $\alpha=2000, \tau=0$, and $\varepsilon=1 \times 10^{-7}$. Likewise, decomposition results of the same signal are illustrated in Figure 8, and the kurtosis values of all IMFs are listed in Table 4.

Following the same ideas, IMF2 with the maximal kurtosis value is chosen and demodulated by Hilbert envelope analysis. The result is presented in Figure 9.

Adopting the proposed method, the maximal kurtosis value of the decomposed IMF listed in Table 2 is 11.06, which is much greater than maximal kurtosis values listed in Tables 3 and 4 . The comparison implies that the sensitive component decomposed by the proposed method shows more obvious fault characteristics. As shown in Figure 4, IMF3 selected by the proposed method demonstrates distinct impact impulses in time-domain waveform. In contrast, mode 4 illustrated in Figure 6 and IMF2 illustrated in Figure 8 both exhibit more noise and less obvious impact impulses. Comparing Figures 5, 7, and 9, it can be observed that, in Figure 5, the fault characteristic frequency of inner raceway $f_{i}$ and its harmonics are highlighted in the envelope spectrum. A fundamental tone at $148.3 \mathrm{~Hz}$ can be clearly found in both Figure 7 and 9, but the higher harmonics like the third and the fourth components are smeared or completely obscured by noise in two envelope spectra. 


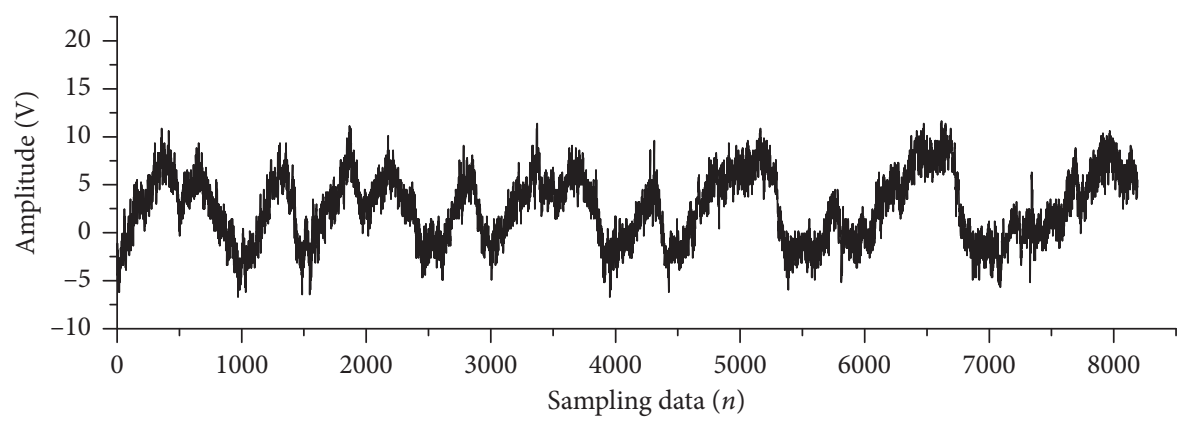

FIGURE 3: A time-domain vibration signal of bearing 6203 with the incipient inner raceway fault.

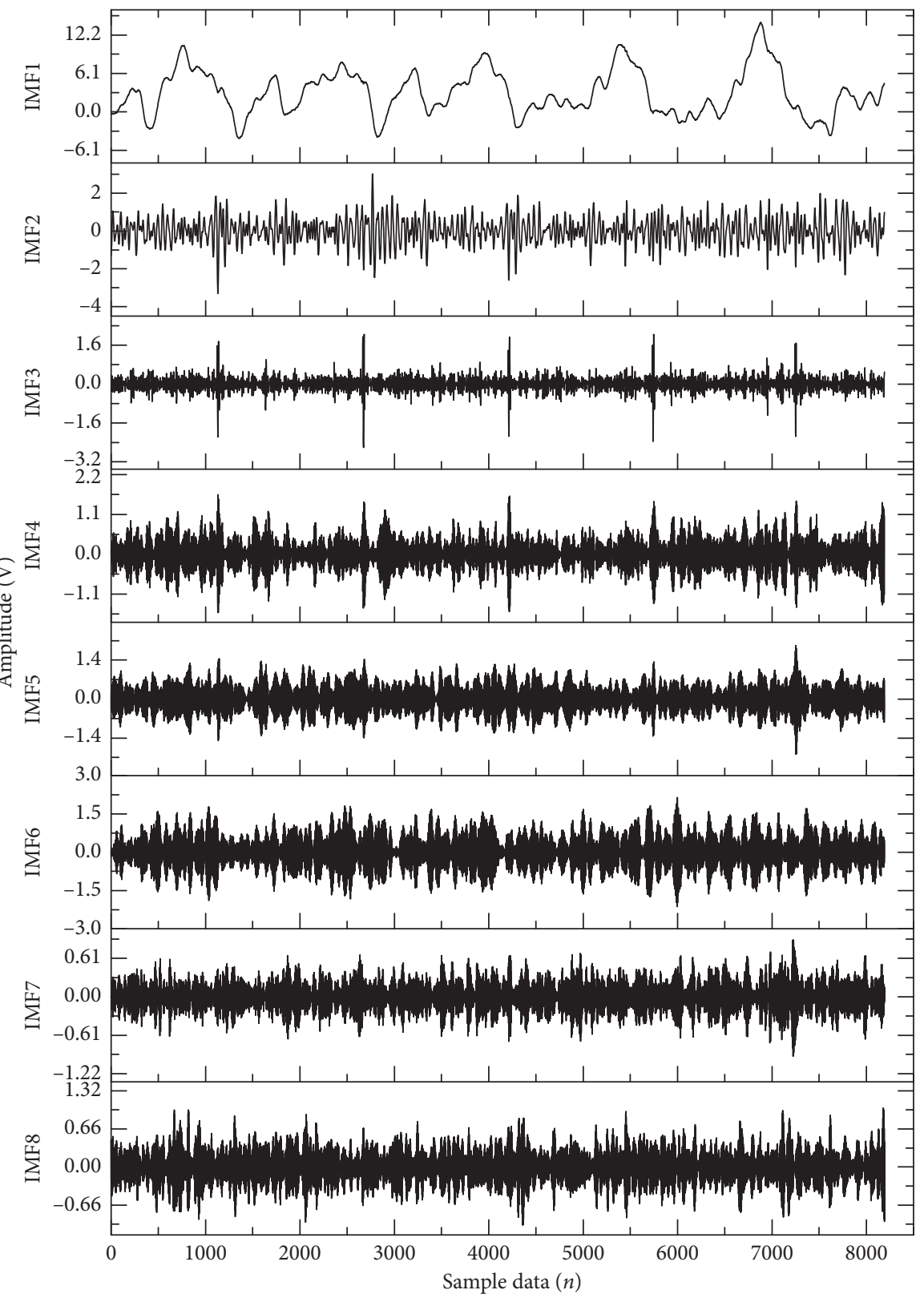

Figure 4: Parameter-adaptive VMD results of the signal with the incipient inner raceway fault.

TABLE 2: Kurtosis values of the decomposed IMFs via parameter-adaptive VMD.

\begin{tabular}{lcccccccc}
\hline IMF & IMF1 & IMF2 & IMF3 & IMF4 & IMF5 & IMF6 & IMF7 & IMF8 \\
\hline$K$ & 2.81 & 3.74 & 11.06 & 3.79 & 2.86 & 2.89 & 3.18 & 3.08 \\
\hline
\end{tabular}




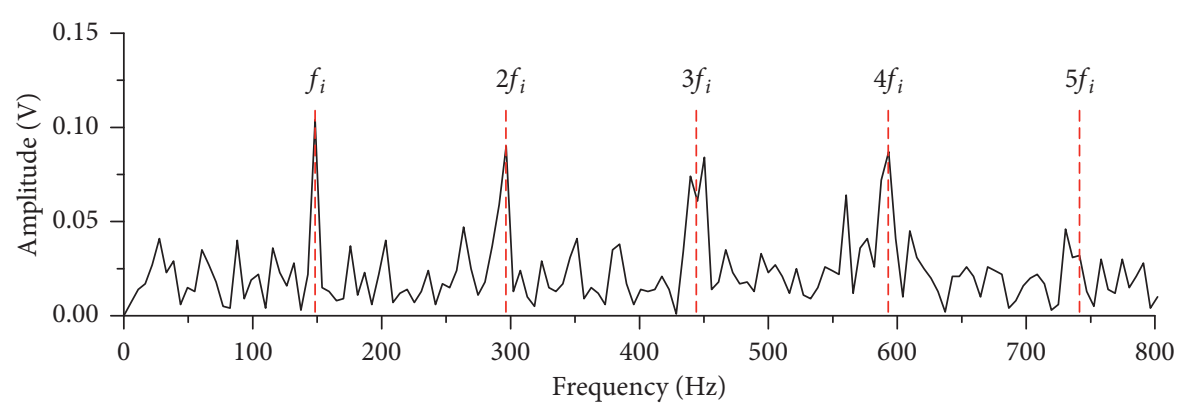

FIGURE 5: Envelope spectrum of the IMF3 decomposed via parameter-adaptive VMD.

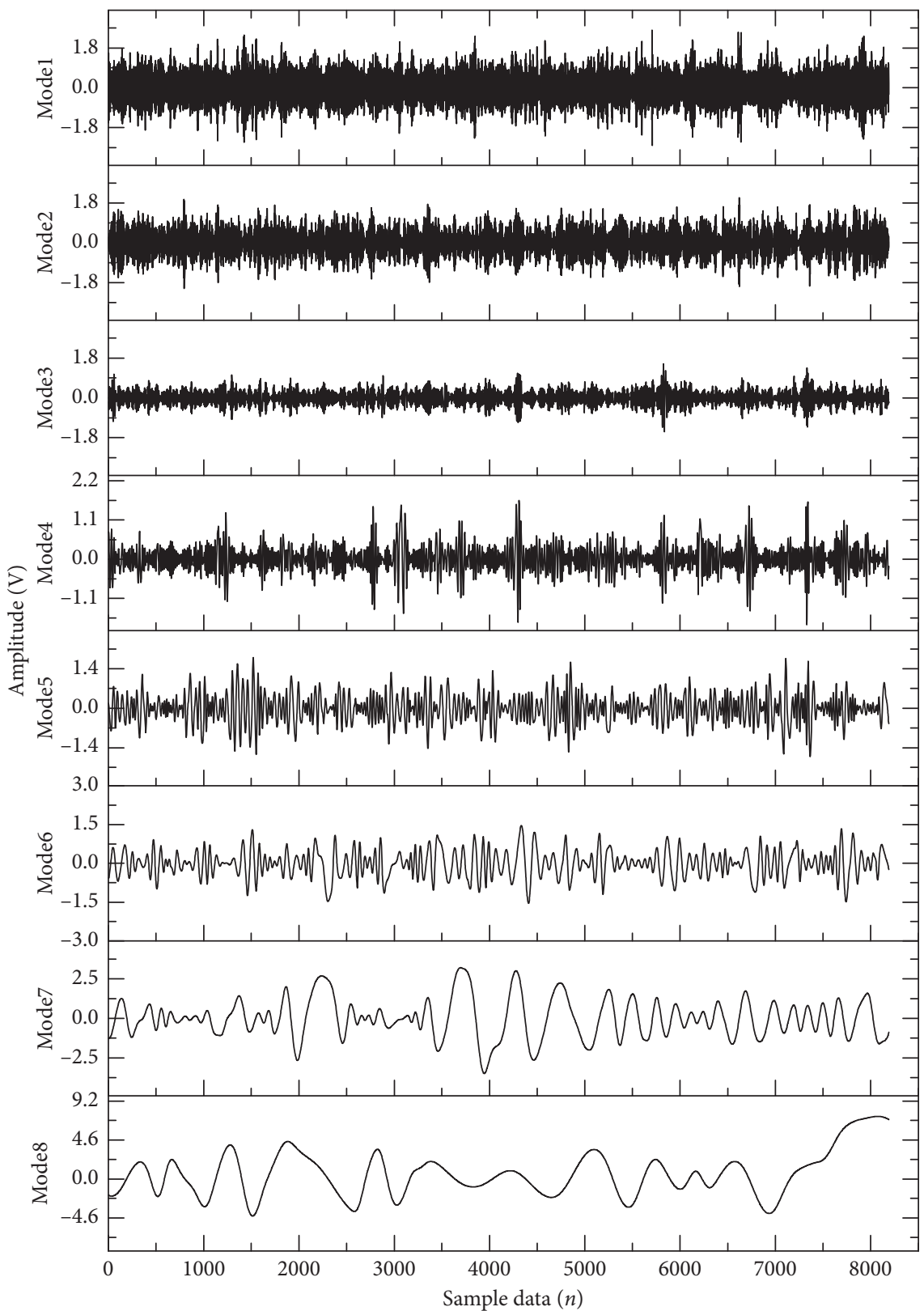

FIGURE 6: EMD results of the vibration signal with the incipient inner raceway fault.

TABLE 3: Kurtosis values of the decomposed modes via EMD.

\begin{tabular}{lcccccccc}
\hline Mode & Mode1 & Mode2 & Mode3 & Mode4 & Mode5 & Mode6 & Mode7 & Mode8 \\
\hline$K$ & 2.11 & 2.85 & 3.73 & 5.17 & 3.46 & 3.04 & 3.12 & 3.30 \\
\hline
\end{tabular}




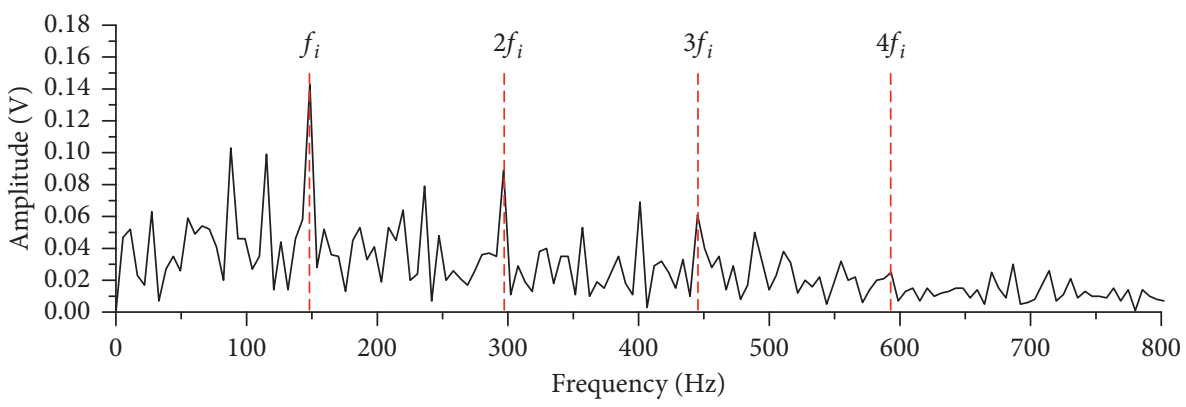

Figure 7: Envelope spectrum of mode4 decomposed via EMD.

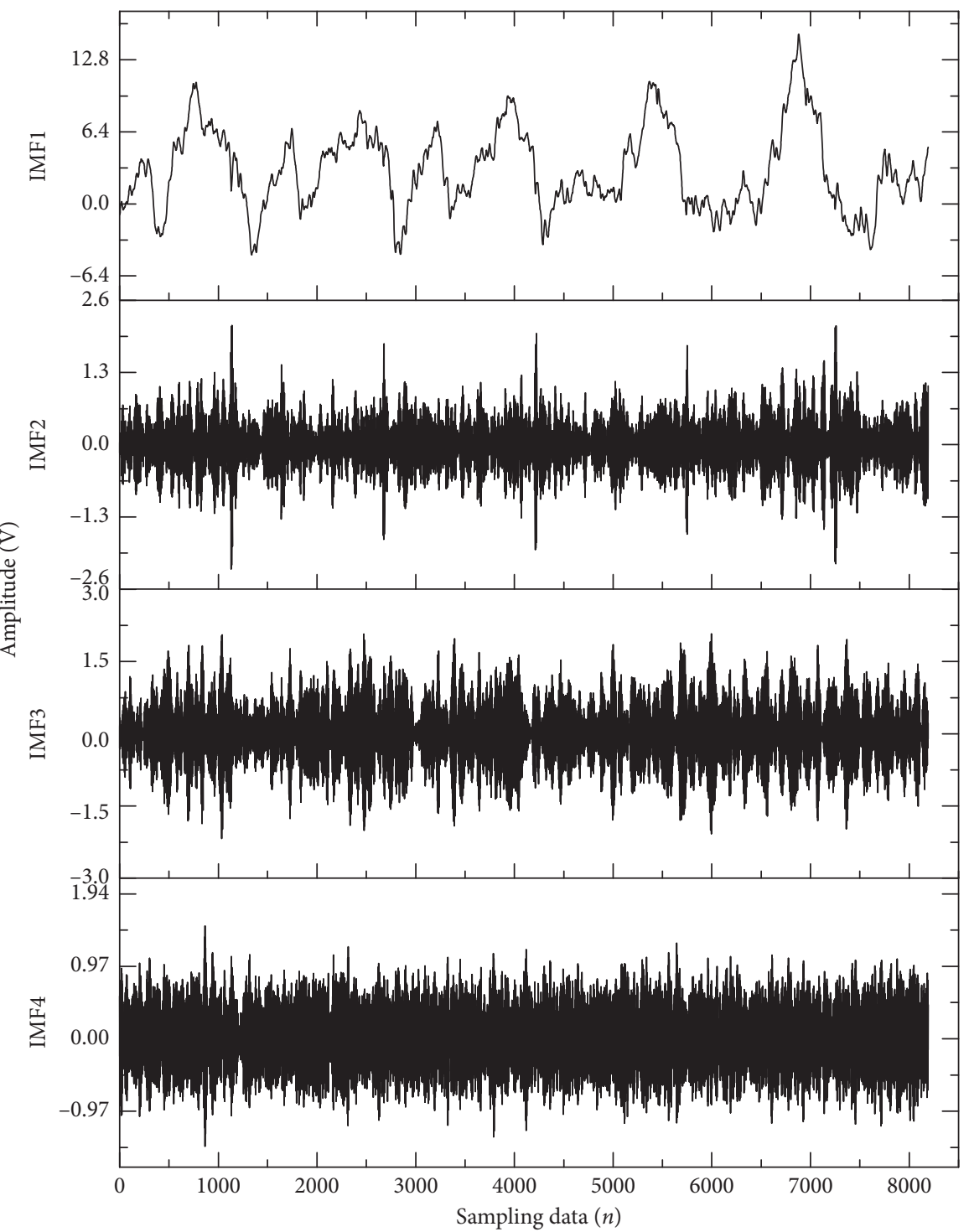

FIgURE 8: Fixed-parameter VMD results of the vibration signal with the incipient inner raceway fault.

TABLE 4: Kurtosis values of the decomposed IMFs via fixed-parameter VMD.

\begin{tabular}{lcccc}
\hline IMF & IMF1 & IMF2 & IMF3 & \\
\hline$K$ & 2.82 & 3.89 & 2.90 & 2.11 \\
\hline
\end{tabular}




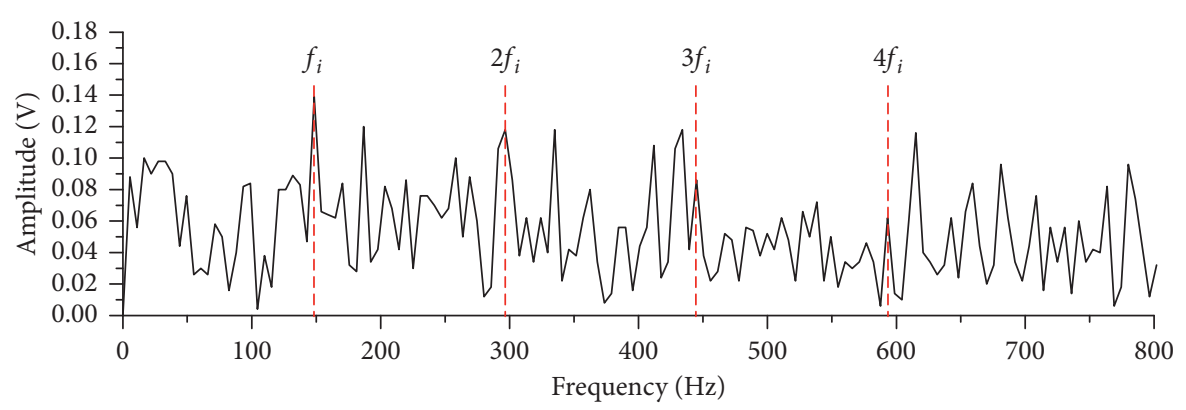

FIGURE 9: Envelope spectrum of IMF2 decomposed via fixed-parameter VMD.

The above experiments demonstrate that the proposed parameter-adaptive VMD outperforms EMD and the fixedparameter VMD with regard to noise robustness and effectiveness in extracting weak fault characteristics contained in bearing vibration signals. Meanwhile, the experiments also indicate the VMD parameters have appreciably impacts on decomposition results. The performance of VMD with inappropriate parameters may be inferior to that of EMD.

4.2. Incipient Outer Raceway Fault. Figure 10 illustrates a time-domain vibration signal with the incipient outer raceway fault. The optimal $M$ and $\alpha$ sought by BAS are 5 and 130 , respectively. Decomposition results of the signal via VMD with the optimal parameters are displayed in Figure 11. The kurtosis values of the resulting IMFs are calculated and listed in Table 5.

As shown in Figure 11, distinct time-domain impulses are exhibited in IMF2, and meanwhile, the maximal kurtosis value appears in IMF2. In view of the aforementioned analysis, IMF2 is separately selected for further envelope analysis via Hilbert transform. The result is displayed in Figure 12.

The fault characteristic frequency of outer raceway $f_{o}$ $(93.4 \mathrm{~Hz})$ and its harmonics have been clearly demonstrated in Figure 12. The distinct spectrum peaks indicate the outer raceway of the bearing is defective.

For comparison, the signal shown in Figure 10 is processed via EMD. Decomposed mode4 with the maximal kurtosis value 10.15 and its envelope spectrum are separately displayed in Figures 13 and 14.

The signal shown in Figure 10 is decomposed by fixedparameter VMD. The key parameters of VMD are the same as those mentioned above, i.e., $M$ and $\alpha$ are set to 4 and 2000, respectively.

IMF2 has the maximal kurtosis value 3.13 in the decomposition results. The time-domain waveform of IMF2 and its corresponding envelope spectrum are separately illustrated in Figures 15 and 16.

Comparing the above experimental results, the following conclusions may be drawn: both parameter-adaptive VMD and EMD can demonstrate clear impact impulses in proper decomposition components, as shown in Figures 11 and 13. The corresponding envelope spectra shown in Figures 12 and 14 highlight the fault characteristic frequency of outer raceway $f_{o}$ and its harmonics, which clearly indicate the outer raceway of the bearing is defective. By contrast, the results decomposed by parameter-adaptive VMD exhibit more prominent and clearer fault characteristic information, while sensitive mode 4 decomposed by EMD involves more noise interference.

It can be observed that IMF2 decomposed by fixedparameter VMD shows little sign of impact impulses but strong noise interference. And it is hard to distinguish the fault characteristic components in the corresponding envelope spectrum displayed in Figure 16. The analysis results indicate that inappropriate parameters of VMD may not identify the fault characteristic frequency.

4.3. Incipient Rolling Element Fault. Figure 17 illustrates a time-domain vibration signal with the incipient rolling element fault. The optimal $M$ and $\alpha$ sought by BAS are 6 and 170 , respectively. Using parameter-adaptive VMD, the vibration signal is processed to acquire the IMF components, and the results are displayed in Figure 18. The kurtosis values of the resulting IMFs are calculated and listed in Table 6.

The envelope spectrum of IMF2 with maximal kurtosis value is displayed in Figure 19. As shown, the raw vibration signal is contaminated by heavy noise, and the weak fault characteristics are buried by other interference frequency components. Nevertheless, satisfactory results can be acquired by the proposed method. As illustrated in Figure 19, the fault characteristic frequency of rolling element $f_{b}$ $(126.3 \mathrm{~Hz})$ along with its second and third harmonics can be easily recognized.

The signal shown in Figure 17 is processed by EMD, in which decomposed mode4 has the maximal kurtosis value 10.73. Mode4 and its envelope spectrum are displayed in Figures 20 and 21, respectively.

Comparing Figure 21 with Figure 19, it can be noted that the fault characteristic frequency $f_{b}$ acquired by EMD may be roughly identified, but the envelope spectrum of mode 4 is mixed with a large amount of noise. It may have something to do with mode mixing effects in the EMD method.

Figure 22 illustrates the time-domain waveform of IMF2 with the maximal kurtosis value 3.06, which is decomposed by fixed-parameter VMD. The envelope spectrum of IMF2 is illustrated in Figure 23.

It is difficult to identify the fault characteristic components in the envelope spectrum shown in Figure 23. The existence of serious mode mixing leads to the selected IMF 


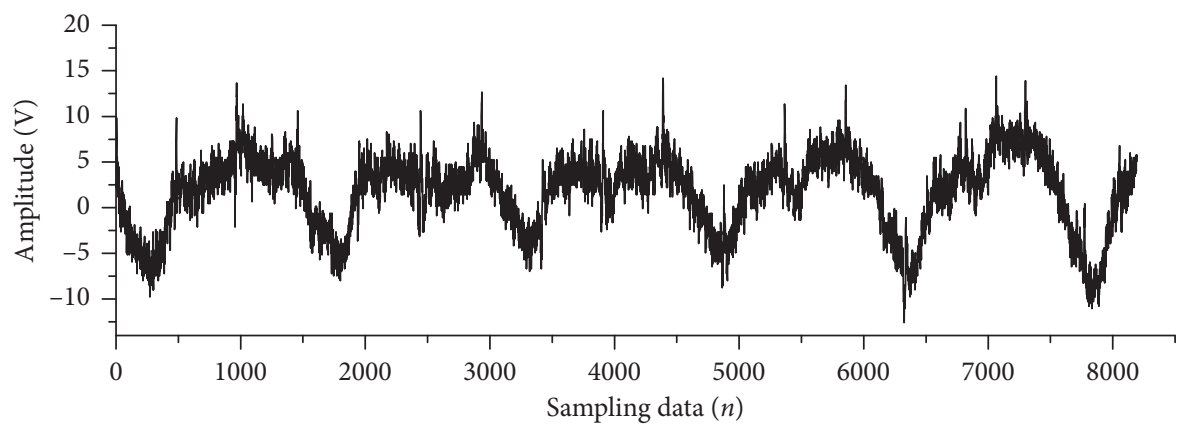

FIgURE 10: Time-domain vibration signal of bearing 6203 with the incipient outer raceway fault.

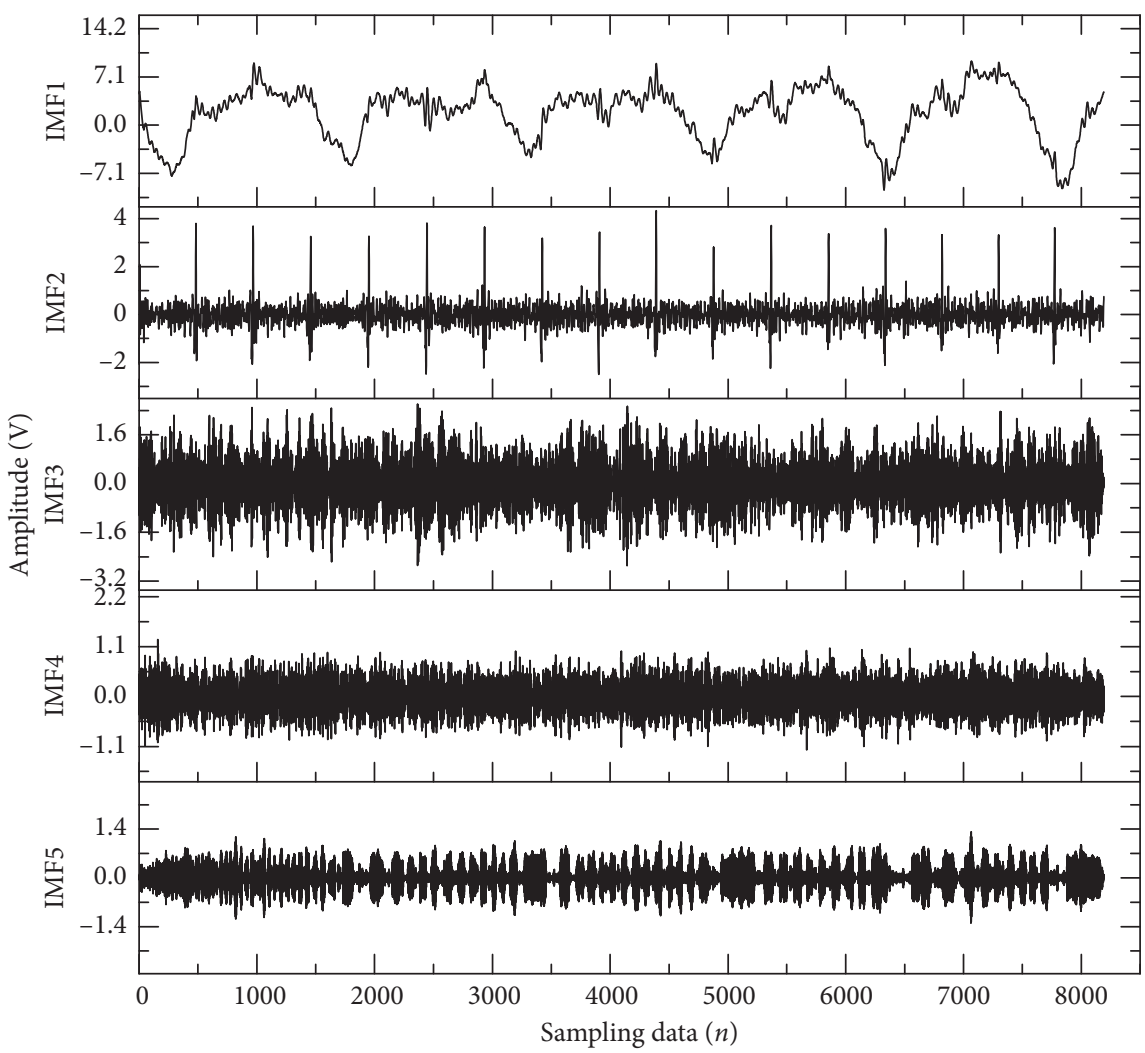

FIgURE 11: Parameter-adaptive VMD results of the vibration signal with the incipient outer raceway fault.

TABLE 5: Kurtosis values of the decomposed IMFs via parameter-adaptive VMD.

\begin{tabular}{lccccc}
\hline IMF & IMF1 & IMF2 & IMF3 & IMF4 & IMF5 \\
\hline$K$ & 2.90 & 15.49 & 2.92 & 2.45 & 2.23 \\
\hline
\end{tabular}

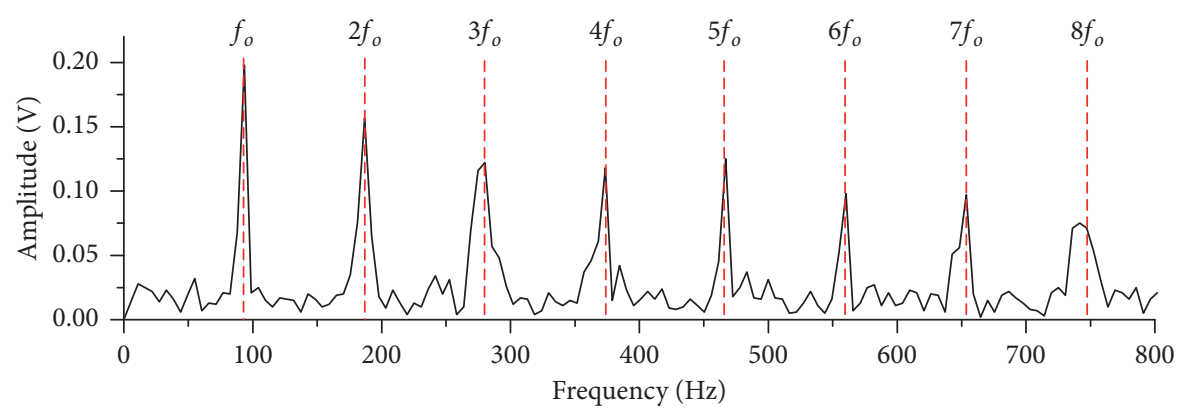

FIGURE 12: Envelope spectrum of IMF2 decomposed via parameter-adaptive VMD. 


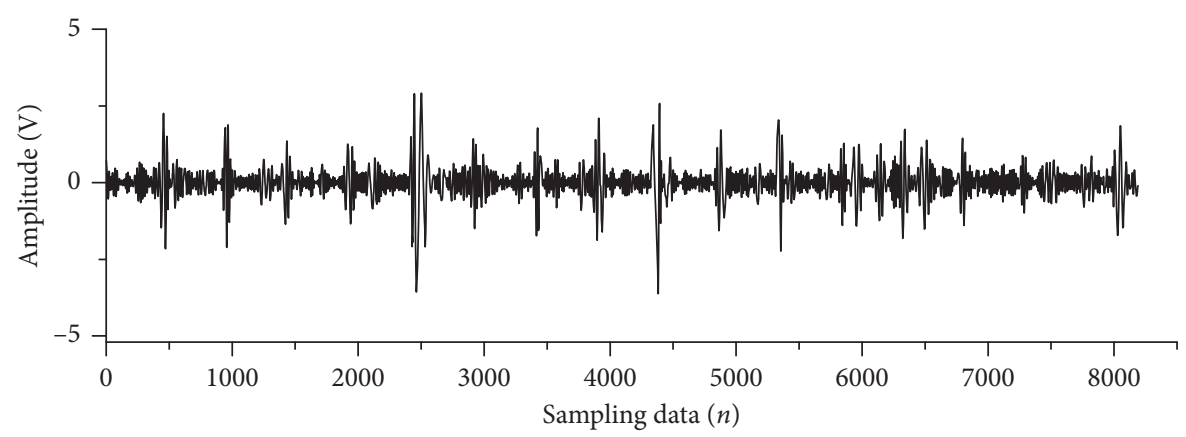

FIGURE 13: Decomposed mode4 obtained by EMD from the incipient outer raceway fault signal.

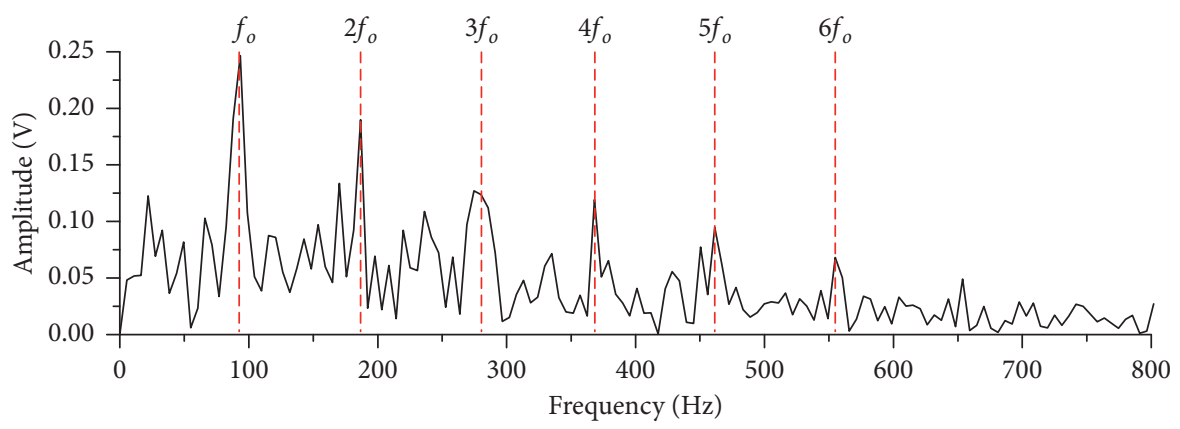

Figure 14: Envelope spectrum of mode4 obtained by EMD.

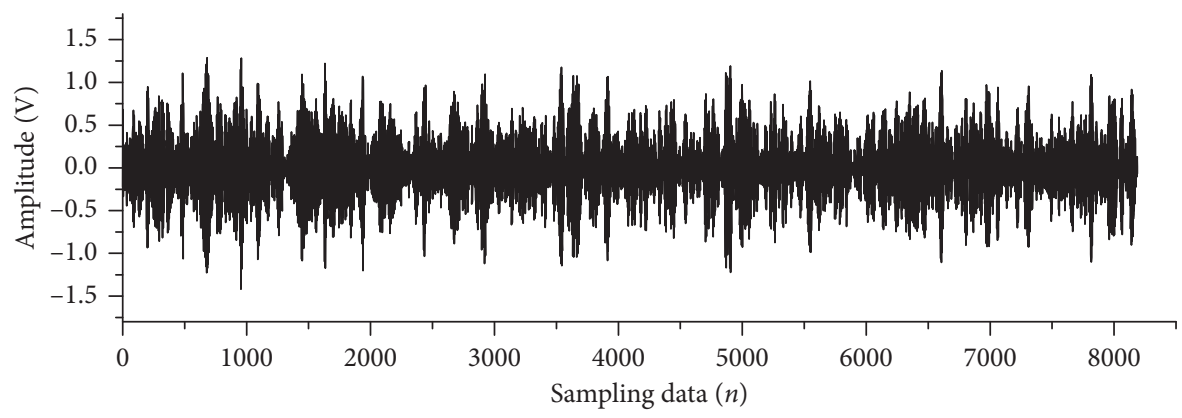

FIGURE 15: Decomposed IMF2 obtained by fixed-parameter VMD from the incipient outer raceway fault signal.

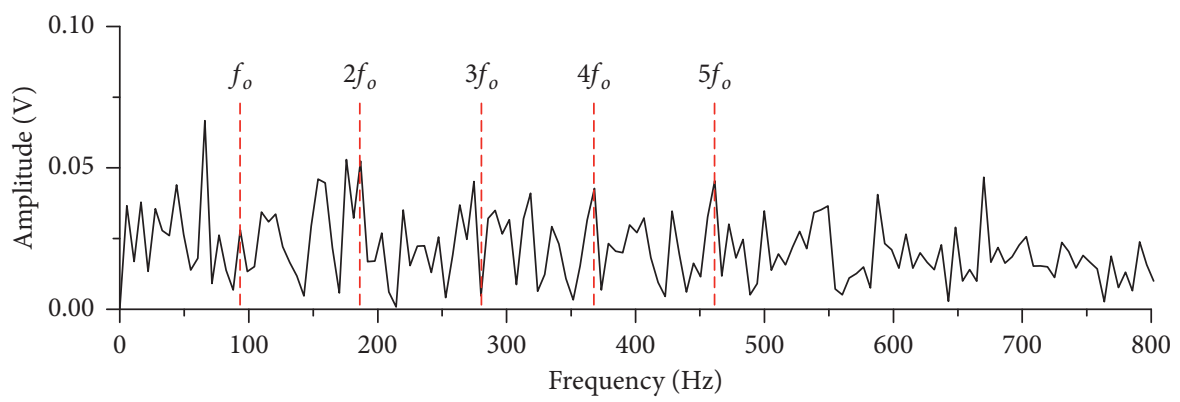

FIGURE 16: Envelope spectrum of IMF2 obtained by fixed-parameter VMD.

containing heavy noise and other frequency components. The strong interference masks the useful fault characteristic information and results in the failure of bearing incipient fault diagnosis.

For further comparison, an extreme learning machine (ELM) [29-31] classifier is adopted to estimate the proposed method. Considering that the energy at the fault characteristic frequencies will increase if the bearing is faulty, the proportion of fault energy (PFE) is firstly introduced. As aforementioned, the measured fault characteristic frequencies may be slightly different from the theoretical ones in the envelope spectrum. Therefore, the calculated fault 


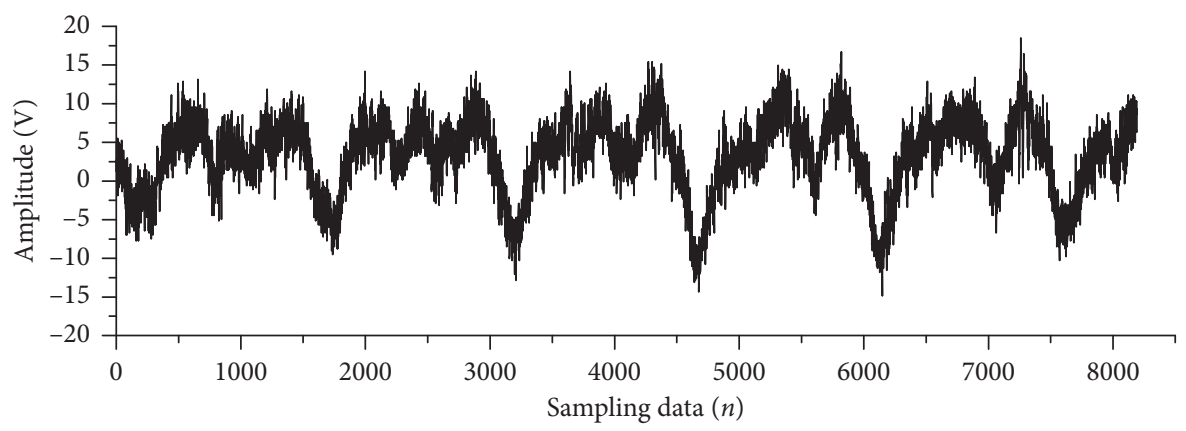

Figure 17: Time-domain vibration signal of bearing 6203 with the incipient rolling element fault.

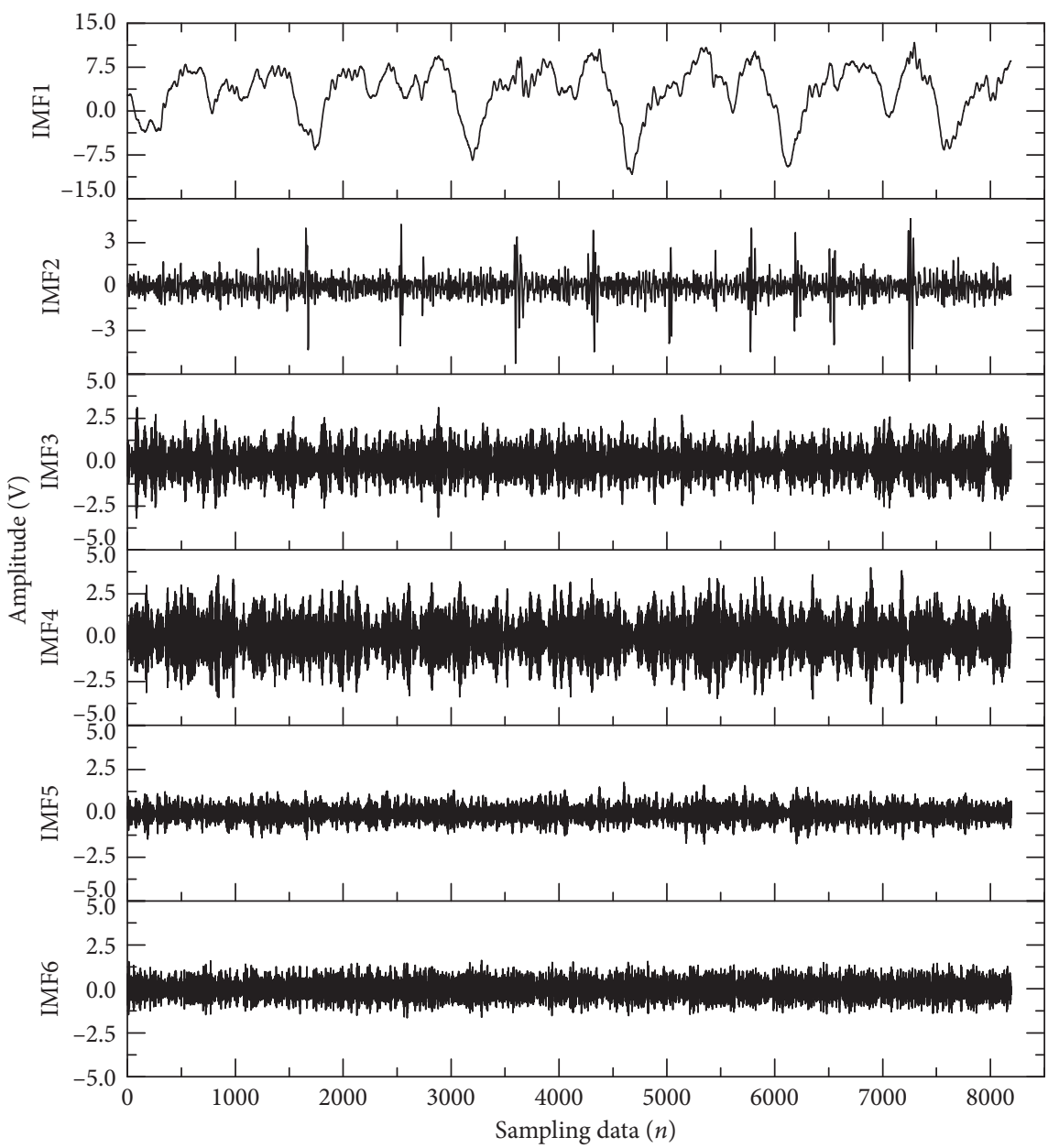

FIgURE 18: Parameter-adaptive VMD results of the vibration signal with the incipient rolling element fault.

TABLE 6: Kurtosis values of the decomposed IMFs via parameteradaptive VMD.

\begin{tabular}{lcccccc}
\hline IMF & IMF1 & IMF2 & IMF3 & IMF4 & IMF5 & IMF6 \\
\hline$K$ & 3.41 & 12.68 & 3.04 & 2.87 & 3.14 & 2.55 \\
\hline
\end{tabular}

characteristic frequency $f$ is updated to a narrow band frequency range $[f-\Delta f, f+\Delta f]$, where $\Delta f$ is set to $5 \mathrm{~Hz}$. The fault energy $E_{1}$ can be calculated by the following equation:

$$
E_{1}=\sum_{j=f-5}^{f+5} f_{j}^{2}
$$

The frequency range $[0.5 f, 1.5 f]$ is chosen to calculate the signal energy $E_{1 a}$ to avoid the influences derived from other frequency bands.

$$
E_{1 a}=\sum_{k=0.5 f}^{1.5 f} f_{k}^{2}
$$




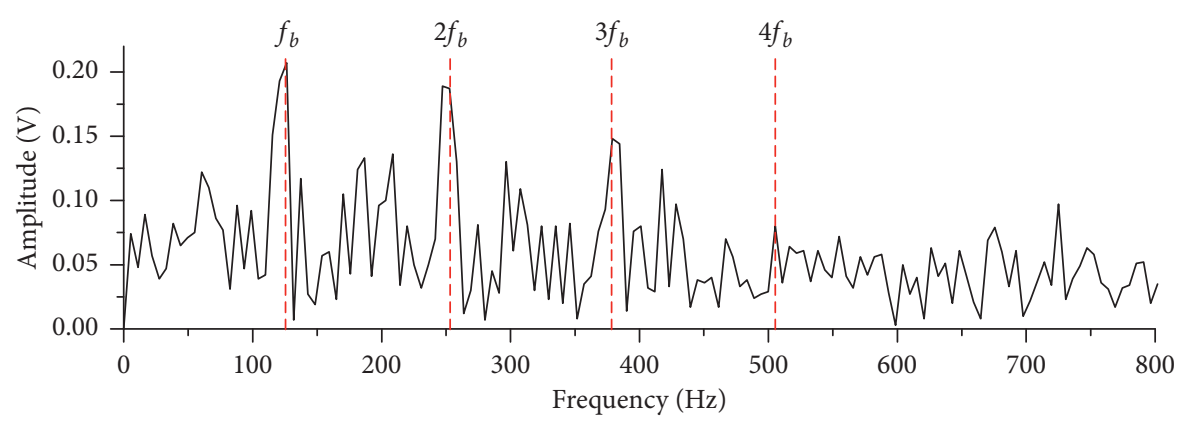

Figure 19: Envelope spectrum of IMF2 decomposed via parameter-adaptive VMD.

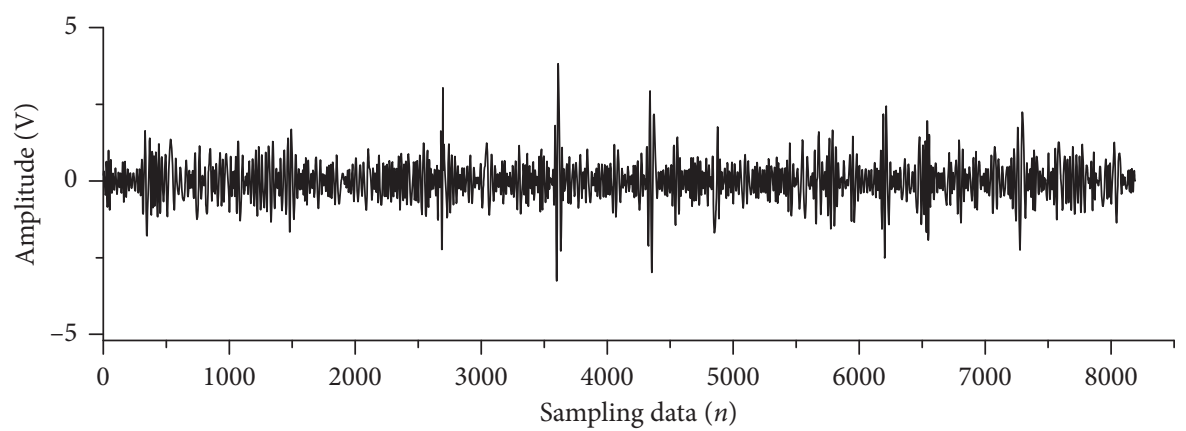

Figure 20: Decomposed mode4 obtained by EMD from the incipient rolling element fault signal.

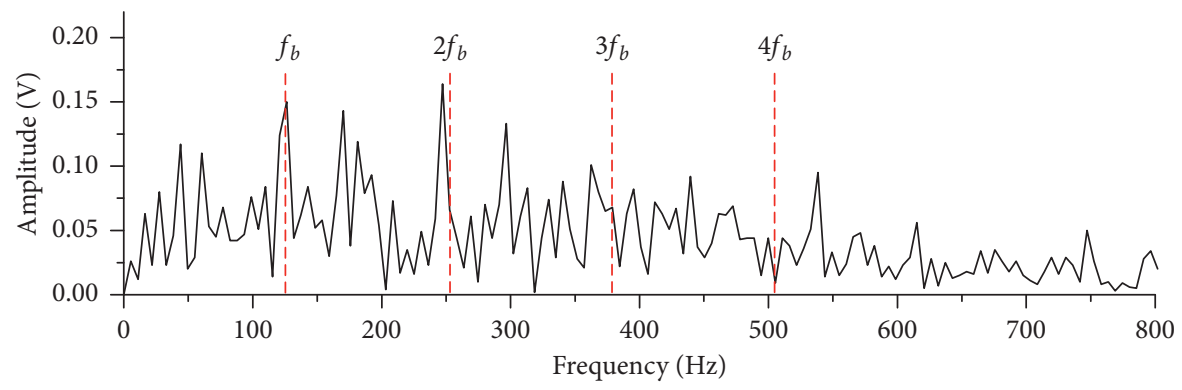

FIGURE 21: Envelope spectrum of mode4 obtained by EMD.

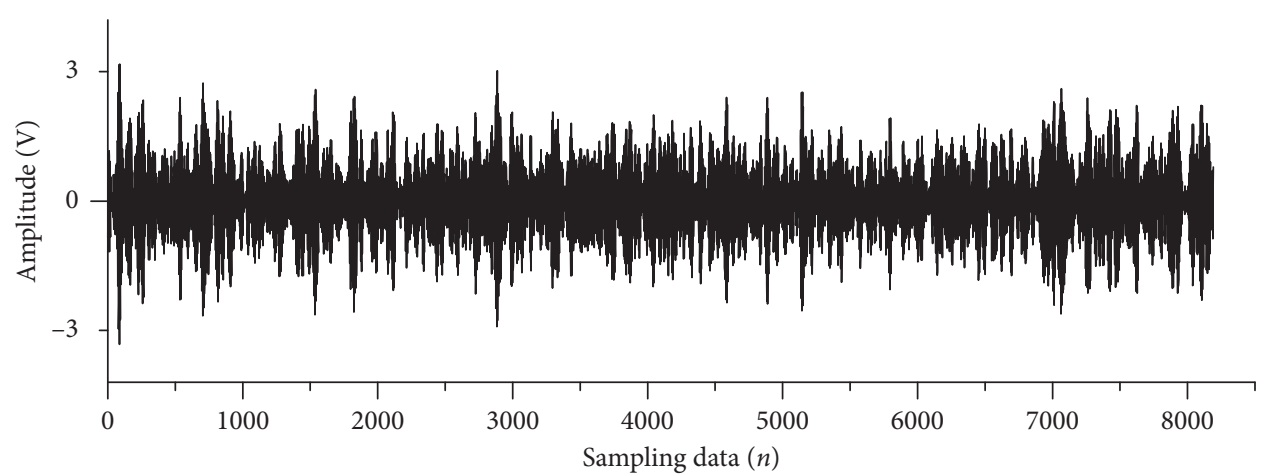

FIGURE 22: Decomposed IMF2 obtained by fixed-parameter VMD from the incipient rolling element fault signal.

The ratio of $R_{1}=E_{1} / E_{1 a}$ is calculated to evaluate the proportion of the 1st harmonic fault energy. Similarly, the 2nd harmonic fault energy $E_{2}$ is calculated. Considering that the deviation between the theoretical 2nd harmonic and the measured one will increase further, the 2 nd harmonic $2 f$ is updated to $[2 f-2 \Delta f, 2 f+2 \Delta f]$, where $\Delta f$ is still set to $5 \mathrm{~Hz}$. 


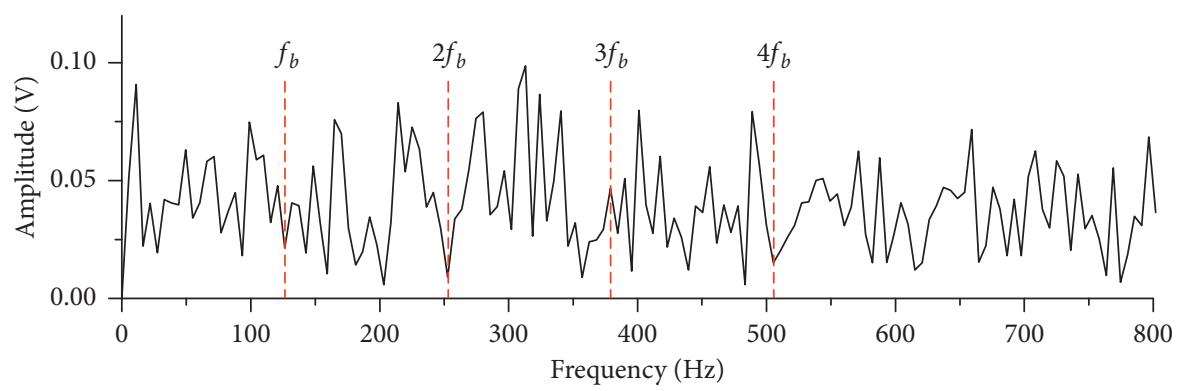

FIgURE 23: Envelope spectrum of IMF2 obtained by fixed-parameter VMD.

TABLE 7: The PFEs obtained by different methods.

\begin{tabular}{|c|c|c|c|c|c|c|c|c|c|}
\hline \multirow{2}{*}{ Methodology } & \multicolumn{3}{|c|}{ Inner raceway fault $f_{i}$} & \multicolumn{3}{|c|}{ Outer raceway fault $f_{o}$} & \multicolumn{3}{|c|}{ Rolling element fault $f_{b}$} \\
\hline & $R_{1 i}$ & $R_{2 i}$ & $R_{1 i}+R_{2 i}$ & $R_{1 o}$ & $R_{2 o}$ & $R_{1 o}+R_{2 o}$ & $R_{1 b}$ & $R_{2 b}$ & $R_{1 b}+R_{2 b}$ \\
\hline The proposed method & 0.496 & 0.497 & 0.993 & 0.902 & 0.924 & 1.826 & 0.395 & 0.402 & 0.797 \\
\hline EMD & 0.256 & 0.268 & 0.524 & 0.571 & 0.425 & 0.996 & 0.290 & 0.355 & 0.645 \\
\hline Fixed-parameter VMD & 0.151 & 0.263 & 0.414 & 0.069 & 0.461 & 0.530 & 0.058 & 0.088 & 0.146 \\
\hline
\end{tabular}

TABLE 8: The testing results of ELM.

\begin{tabular}{lcccccc}
\hline \multirow{2}{*}{ Methodology } & & \multicolumn{2}{c}{ Testing error } & & Testing accuracy \\
& Normal & Inner raceway fault & Outer raceway fault & Rolling element fault & Total & $9 / 80$ \\
\hline The proposed method & $2 / 20$ & $3 / 20$ & $1 / 20$ & $0 / 20$ & $92.5 \%$ \\
EMD & $2 / 20$ & $5 / 20$ & $2 / 20$ & $0 / 20$ & $9 / 80$ & $88.75 \%$ \\
Fixed-parameter VMD & $4 / 20$ & $9 / 20$ & $5 / 20$ & $5 / 20$ & $23 / 80$ & $71.25 \%$ \\
\hline
\end{tabular}

$$
E_{2}=\sum_{j=2 f-10}^{2 f+10} f_{j}^{2}
$$

The frequency range $[1.5 f, 2.5 f]$ is chosen to calculate the signal energy $E_{2 a}$ :

$$
E_{2 a}=\sum_{k=1.5 f}^{2.5 f} f_{k}^{2}
$$

The ratio of $R_{2}=E_{2} / E_{2 a}$ is calculated to evaluate the proportion of the 2 nd harmonic fault energy. The deviation between the theoretical 3rd harmonic and the measured one will continue to increase, which may introduce a significant error. Therefore, the proportion of the 3rd harmonic fault energy is no longer calculated. The PFEs of the envelope spectra above are calculated and listed in Table 7.

As displayed in Table 7, the PFEs obtained by the proposed method are higher than those obtained by the other two methods, which is beneficial for incipient fault diagnosis of bearing.

In this study, $K, R_{1 i}+R_{2 i}, R_{1 o}+R_{2 o}$, and $R_{1 b}+R_{2 b}$ are calculated and chosen as the input vector of ELM. Four states of vibration signals, separately corresponding to the normal state, inner raceway fault, outer raceway fault, and rolling element fault, are assigned as the output vector of ELM. There are four groups of signals corresponding to the four states. Each of four groups involves 20 training signals and 20 testing signals. The hidden layer nodes of ELM are set to 80. The testing results of ELM are listed in Table 8.
As shown in Table 8, the fault diagnosis accuracy of the proposed method is higher than that of the other two methods. Compared with the proposed method and EMD, the testing accuracy of the fixed-parameter VMD is much lower, which indicates that $M$ and $\alpha$ will greatly affect the VMD results.

\section{Conclusions}

The VMD method can completely decompose the raw vibration signals into a set of IMFs from low frequency to high frequency. The mode number $M$ and the quadratic penalty term $\alpha$ of VMD will have considerable influence on decomposition results. The key to the VMD method lies in seeking for the optimal combination of $M$ and $\alpha$ that matches with the analyzed signals.

In this paper, the BAS optimization algorithm is employed to adaptively estimate the optimal $M$ and $\alpha$. The optimized parameters can guarantee the availability of VMD.

The proposed parameter-adaptive VMD method has been applied in the field tests for low-noise deep groove ball bearing 6203. Comparisons have also been conducted to evaluate the performances by using the proposed method, EMD, and the fixed-parameter VMD. Three cases studies demonstrate that the proposed method outperforms EMD and the fixed-parameter VMD in suppressing the noise interference and highlighting the weak fault characteristic frequency information contaminated by heavy noise. 


\section{Data Availability}

The data used to support the findings of this study are available from the corresponding author upon request.

\section{Conflicts of Interest}

The authors declare that they have no conflicts of interest.

\section{References}

[1] S. P. Mogal and D. I. Lalwani, "A brief review on fault diagnosis of rotating machineries," Applied Mechanics and Materials, vol. 541-542, pp. 635-640, 2014.

[2] R. Liu, B. Yang, E. Zio, and X. Chen, "Artificial intelligence for fault diagnosis of rotating machinery: a review," Mechanical Systems and Signal Processing, vol. 108, pp. 33-47, 2018.

[3] Q. Gao, H. Tang, J. Xiang, Y. Zhong, S. Ye, and J. Pang, "A Walsh transform-based Teager energy operator demodulation method to detect faults in axial piston pumps," Measurement, vol. 134, pp. 293-306, 2019.

[4] D. Wang, K.-L. Tsui, and Q. Miao, "Prognostics and health management: a review of vibration based bearing and gear health indicators," IEEE Access, vol. 6, pp. 665-676, 2018.

[5] G.-Y. Lee, M. Kim, Y.-J. Quan et al., "Machine health management in smart factory: a review," Journal of $\mathrm{Me}$ chanical Science and Technology, vol. 32, no. 3, pp. 987-1009, 2018.

[6] T. Narendiranath Babu, T. Manvel Raj, and T. Lakshmanan, "A review on application of dynamic parameters of journal bearing for vibration and condition monitoring," Journal of Mechanics, vol. 31, no. 4, pp. 391-416, 2015.

[7] A. Rai and S. H. Upadhyay, "A review on signal processing techniques utilized in the fault diagnosis of rolling element bearings," Tribology International, vol. 96, pp. 289-306, 2016.

[8] I. El-Thalji and E. Jantunen, "A summary of fault modelling and predictive health monitoring of rolling element bearings," Mechanical Systems and Signal Processing, vol. 60-61, no. 1, pp. 252-272, 2015.

[9] Y. Lei, J. Lin, Z. He, and M. J. Zuo, "A review on empirical mode decomposition in fault diagnosis of rotating machinery," Mechanical Systems and Signal Processing, vol. 35, no. 12, pp. 108-126, 2013.

[10] H. Ocak and K. A. Loparo, "Estimation of the running speed and bearing defect frequencies of an induction motor from vibration data," Mechanical Systems and Signal Processing, vol. 18, no. 3, pp. 515-533, 2004.

[11] N. E. Huang, Z. Shen, S. R. Long et al., "The empirical mode decomposition and the Hilbert spectrum for nonlinear and non-stationary time series analysis," Proceedings of the Royal Society of London. Series A: Mathematical, Physical and Engineering Sciences, vol. 454, no. 1971, pp. 903-995, 1998.

[12] N. E. Huang, M.-L. C. Wu, S. R. Long et al., "A confidence limit for the empirical mode decomposition and Hilbert spectral analysis," Proceedings of the Royal Society of London. Series A: Mathematical, Physical and Engineering Sciences, vol. 459, no. 2037, pp. 2317-2345, 2003.

[13] K. Dragomiretskiy and D. Zosso, "Variational mode decomposition," IEEE Transactions on Signal Processing, vol. 62, no. 3, pp. 531-544, 2014.

[14] W. Yang, Z. Peng, K. Wei et al., "Superiorities of variational mode decomposition over empirical mode decomposition particularly in time-frequency feature extraction and wind turbine condition monitoring," IET Renewable Power Generation, vol. 11, no. 4, pp. 443-452, 2016.

[15] Y. Wang, R. Markert, J. Xiang, and W. Zheng, "Research on variational mode decomposition and its application in detecting rub-impact fault of the rotor system," Mechanical Systems and Signal Processing, vol. 60-61, pp. 243-251, 2015.

[16] G. Ren, J. Jia, J. Mei, X. Jia, J. Han, and Y. Wang, "An improved variational mode decomposition method and its application in diesel engine fault diagnosis," Journal of Vibroengineering, vol. 20, no. 6, pp. 2363-2378, 2018.

[17] J. Lian, Z. Liu, H. Wang, and X. Dong, "Adaptive variational mode decomposition method for signal processing based on mode characteristic," Mechanical Systems and Signal Processing, vol. 107, pp. 53-77, 2018.

[18] Y. Liu, J. Wang, Y. Li et al., "Feature extraction method based on VMD and MFDFA for fault diagnosis of reciprocating compressor valve," Journal of Vibroengineering, vol. 19, no. 8, pp. 6007-6020, 2017.

[19] X. Zhang, Q. Miao, H. Zhang, and L. Wang, "A parameteradaptive VMD method based on grasshopper optimization algorithm to analyze vibration signals from rotating machinery," Mechanical Systems and Signal Processing, vol. 108, pp. 58-72, 2018.

[20] Z. Wang, G. He, W. Du et al., "Application of parameter optimized variational mode decomposition method in fault diagnosis of gearbox," IEEE Access, vol. 7, pp. 44871-44882, 2019.

[21] X. Jiang and S. Li, "BAS: beetle antennae search algorithm for optimization problems," International Journal of Robotics and Control, vol. 1, no. 1, 2018.

[22] Z. Liu, H. Cao, X. Chen, Z. He, and Z. Shen, "Multi-fault classification based on wavelet SVM with PSO algorithm to analyze vibration signals from rolling element bearings," Neurocomputing, vol. 99, pp. 399-410, 2012.

[23] H. Yuan, J. Chen, and G. Dong, "bearing fault diagnosis based on improved locality-constrained linear coding and adaptive PSO-optimized SVM," Mathematical Problems in Engineering, vol. 2017, Article ID 7257603, 16 pages, 2017.

[24] S. Wang, J. Xiang, H. Tang, X. Liu, and Y. Zhong, "Minimum entropy deconvolution based on simulation-determined band pass filter to detect faults in axial piston pump bearings," ISA Transactions, vol. 88, pp. 186-198, 2019.

[25] H.-d. Wang, S.-e. Deng, J.-x. Yang, and H. Liao, “A fault diagnosis method for rolling element bearing (REB) based on reducing REB foundation vibration and noise-assisted vibration signal analysis," Proceedings of the Institution of Mechanical Engineers, Part C: Journal of Mechanical Engineering Science, vol. 233, no. 7, pp. 2574-2587, 2019.

[26] Y. Zhang, B. Tang, and X. Xiao, "Time-frequency interpretation of multi-frequency signal from rotating machinery using an improved Hilbert-Huang transform," Measurement, vol. 82, pp. 221-239, 2016.

[27] D. Wang, "Spectral L2/L1 norm: a new perspective for spectral kurtosis for characterizing non-stationary signals," Mechanical Systems and Signal Processing, vol. 104, pp. 290-293, 2018.

[28] C. Yi, J. Lin, W. Zhang, and J. Ding, "Faults diagnostics of railway axle bearings based on IMF's confidence index algorithm for ensemble EMD," Sensors, vol. 15, no. 5, pp. 10991-11011, 2015.

[29] G. B. Huang, Q. Y. Zhu, and C. K. Siew, "Extreme learning machine: a new learning scheme of feedforward neural networks," in Proceedings of the 2004 IEEE International Joint Conference on Neural Networks, pp. 985-990, Institute of 
Electrical and Electronics Engineers Inc., Piscataway, NJ, USA, July 2004.

[30] G. B. Huang, Q. Y. Zhu, and C. K. Siew, "Extreme learning machine: theory and applications," Neurocomputing, vol. 70, no. $1-3$, pp. 489-501, 2006.

[31] M. Liang, D. Su, D. Hu et al., "A novel faults diagnosis method for rolling element bearings based on ELCD and extreme learning machine," Shock and Vibration, vol. 2018, Article ID 1891453, 10 pages, 2018. 